\title{
Accurate Dissociation Energies of Two Isomers of the 1-Naphthol·Cyclopropane Complex
}

\author{
Surajit Maity ${ }^{1,3}$, Richard Knochenmuss ${ }^{1}$, Christof Holzer ${ }^{2}$, Géraldine \\ Féraud $^{1,4}$, Jann Frey ${ }^{1}$, Wim Klopper ${ }^{2}$ and Samuel Leutwyler ${ }^{1}$ \\ ${ }^{1}$ Departement für Chemie und Biochemie, Universität Bern, \\ Freiestrasse 3, CH-3000 Bern 9, Switzerland* and \\ ${ }^{2}$ Karlsruher Institut für Technologie (KIT), Institut für Physikalische Chemie, \\ Fritz-Haber-Weg 2, D-76131 Karlsruhe, Germany ${ }^{\dagger}$
}

(Dated: October 6, 2016)

\begin{abstract}
The 1-naphthol-cyclopropane intermolecular complex is formed in a supersonic jet and investigated by resonant two-photon ionization (R2PI) spectroscopy, UV holeburning, and stimulated emission pumping (SEP)-R2PI spectroscopy. Two very different structure types are inferred from the vibronic spectra and calculations. In the "edge" isomer, the $\mathrm{OH}$ group of 1-naphthol is directed towards a C-C bond of cyclopropane, the two ring planes are perpendicular. In the "face" isomer, the cyclopropane is adsorbed on one of the $\pi$-aromatic faces of the 1-naphthol moiety, the ring planes are nearly parallel. Accurate ground-state intermolecular dissociation energies $D_{0}$ were measured with the SEP-R2PI technique. The $D_{0}\left(S_{0}\right)$ of the edge isomer is bracketed as $15.35 \pm 0.03 \mathrm{~kJ} / \mathrm{mol}$, while that of the face isomer is $16.96 \pm 0.12 \mathrm{~kJ} / \mathrm{mol}$. The corresponding excited-state dissociation energies $D_{0}\left(S_{1}\right)$ were evaluated using the respective electronic spectral shifts. Despite the $D_{0}\left(S_{0}\right)$ difference of $1.6 \mathrm{~kJ} / \mathrm{mol}$, both isomers are observed in the jet in similar concentrations, so they must be separated by substantial potential energy barriers. Intermolecular binding energies, $D_{e}$, and dissociation energies, $D_{0}$, calculated with correlated wave function methods and two dispersion-corrected density-functional methods are evaluated in the context of these results. The density functional calculations suggest the face isomer is bound solely by dispersion interactions. Binding of the edge isomer is also dominated by dispersion, which makes up two thirds of the total binding energy.
\end{abstract}

Keywords: dispersive interactions, non classical hydrogen bond, intermolecular binding energies, laser spectroscopy 


\section{INTRODUCTION}

Intermolecular interactions of aliphatic $\mathrm{C}$-H groups with aromatic $\pi$-electron systems on the one hand and of polar $\mathrm{X}-\mathrm{H}$ moieties with aliphatic $\mathrm{C}-\mathrm{C}$ and $\mathrm{C}=\mathrm{C}$ bonds on the other hand have been denoted weak hydrogen bonds, non-classical hydrogen bonds or C-H/ $\pi$ interactions. ${ }^{1-3}$ In spite of their weakness, non-classical hydrogen bonds have been frequently invoked in systems from macromolecules to biomolecules to explain physical and chemical properties such as crystal structures, conformations and reactivity. ${ }^{1,2,4}$ The detailed nature of such interactions remains insufficiently understood, and may include aspects such as charge transfer contributions from the $\pi$ system to C-H anti-bonding orbitals, as well as London dispersion. ${ }^{5-8}$ Deeper understanding will benefit from quantitative characterization of systems such as the bimolecular complexes presented here.

The ground state binding energy, $D_{e}\left(S_{0}\right)$, and dissociation energy, $D_{0}\left(S_{0}\right)$, (see Figure 1) are among the fundamental parameters characterizing intermolecular interactions. ${ }^{9}$ However, accurate experimental dissociation energies in the gas phase, free from other interactions and perturbing solvent effects, have only been measured for a few dozen intermolecular complexes, for a recent review see Ref. 9. Among these are van der Waals complexes M.S of aromatic chromophores M such as benzene, ${ }^{10}$ para-difluorobenzene, ${ }^{11,12}$ phenol, ${ }^{13}$ anisole,${ }^{14}$ indole, ${ }^{15}$ dibenzo-p-dioxin, ${ }^{16}$ and carbazole ${ }^{17-19}$ with noble gases $(\mathrm{S}=\mathrm{Ne}, \mathrm{Ar}, \mathrm{Kr}, \mathrm{Xe})$, or small closed-shell molecules $\left(\mathrm{S}=\mathrm{N}_{2}\right.$, $\left.\mathrm{CO}, \mathrm{CH}_{4}\right) .{ }^{19}$ In these cases, linear correlation of $D_{0}\left(S_{0}\right)$ with the polarizability of the $\mathrm{S}$ atom or molecule was observed, as expected from London dispersion theory. ${ }^{9}$ Larger $\mathrm{CH} \cdots \pi$ complexes, such as benzene-alkanes, were measured using a two-color appearance potential method..$^{20,21}$ Again a linear correlation with the average molecular polarizabilities was found. ${ }^{6,7}$

Currently, we are extending measurement of $D_{0}\left(S_{0}\right)$ values to more complex and chemically relevant cases such as 1-naphthol $(1-\mathrm{NpOH})$ with hydrocarbons. In these complexes the 1-NpOH can act as a non classical H-bond donor to the alkane, or the alkane can act as a $\mathrm{CH} / \pi$ donor. While the dissociation energies of 1-naphthol-benzene and 1-naphthol-cyclohexane have been measured, ${ }^{22}$ their structures are not known, and both electrostatic and dispersion interactions may be significant. We show here that in $1-\mathrm{NpOH} \cdot$ cyclopropane both naphthol and cyclopropane act as a non classical H-bond donors and acceptors.

Because of its strained three-membered ring, some properties of cyclopropane are closer to those of alkenes than alkanes. ${ }^{23,24}$ Its equilibrium $\mathrm{C}-\mathrm{C}$ bond length of $1.503 \AA$ is shorter than that 
of $n$-alkanes, indicating a slight double-bond character. ${ }^{23,25}$ In crystal structures listed in the Cambridge database $\mathrm{CSD},{ }^{26}$ the cyclopropyl moiety occurs both as an $\mathrm{H}$-bond acceptor to the C-C bond and as a $\mathrm{CH}$ donor. ${ }^{24}$ The $\mathrm{CSD}$ database analyses suggest that cyclopropane is a better $\mathrm{CH}$ donor than $n$-alkanes and slightly better than alkenes. ${ }^{24}$ However, gas-phase spectroscopic evidence of $\mathrm{H}$-bonded cyclopropane is rather sparse. ${ }^{27-29} \mathrm{~A}$ paradigmatic example is the F-H.cyclopropane complex, in which the HF moiety forms an H-bond to the C-C bond of cyclopropane. ${ }^{29}$

In 1-NpOH·cyclopropane, a structure can occur with cyclopropane adsorbed to one face of the naphthalene $\pi$-system, giving rise to $\mathrm{CH} \cdots \pi$ interactions. On the other hand, the $\mathrm{OH}$ group of 1- $\mathrm{NpOH}$ may act as an $\mathrm{H}$-donor to a $\mathrm{C}-\mathrm{C}$ bond of cyclopropane, analogous to the structure of F-H.cyclopropane. ${ }^{29}$ Below, we show that both isomers are formed; we can determine the $D_{0}\left(S_{0}\right)$ values of both isomers separately to within $\pm 3 \mathrm{~cm}^{-1}$ and $\pm 10 \mathrm{~cm}^{-1}$, making these measurements among the most accurate experimental intermolecular dissociation energies to date. ${ }^{9,30-32}$

These measurements on isomers of the same complex represent useful benchmarks and a special challenge for theory. The accuracy of calculated dissociation energies of large intermolecular complexes depend on whether the structure is $\pi$-stacked or H-bonded. Some methods tend to be more accurate for the H-bonded structures (MP2, MP2-R12), others for the $\pi$-stacked systems (SCS-MP2 and SCS-MP2-R12). ${ }^{33-35}$ In a recent comparison of calculated and experimental $D_{0}$ values for eleven H-bonded and eleven dispersion-bound complexes, Haldar et al. calculated the H-bonded complexes with the MP2 method, but the dispersive complexes using the B97-D3 density functional. ${ }^{36}$ While the mean relative deviations of the calculations were reasonable $(15.1 \%$ with MP2 and 7.7\% with B97-D3), it is heuristically unsatisfactory to select the computational method based on the presumed type of intermolecular interaction.

\section{METHODS}

\section{A. The SEP-R2PI technique}

The ground-state dissociation energies of jet-cooled intermolecular $\mathrm{M} \cdot \mathrm{S}$ ( $\mathrm{S}=$ solvent) complexes were determined using a vibrational predissociation process, ${ }^{9,37}$ in which the $S_{0}$ state vibrational levels are populated using stimulated-emission pumping (SEP) ${ }^{38}$ via the $S_{0} \leftrightarrow S_{1}$ transition of the chromophore $\mathrm{M}$; the vibrational predissociation of the hot $\mathrm{M} \cdot \mathrm{S}$ levels is detected by resonant two photon ionization (R2PI). This triply-resonant method is abbreviated as SEP-R2PI,,$^{9,17-19,22,38-41}$ a 
scheme is shown in Figure 1.

First, a pump laser (1), fixed at the $S_{0} \rightarrow S_{1}$ origin of the aromatic chromophore M, excites the jet-cooled M.S complex to the vibrationless $S_{1}$ state. After a small delay $(\Delta t=1-2 \mathrm{~ns})$, the dump laser (2) is scanned at photon energies smaller than the pump laser. When it is resonant with a vibrationally excited ground state, $S_{0}^{\neq}$, some excited-state population is transferred to the "hot" $S_{0}^{\neq}$level by stimulated emission. After $1.1 \mu$ s time delay that is long enough for the $\mathrm{M}^{\neq} \cdot \mathrm{S}$ complex to undergo intramolecular vibrational redistribution (IVR) and, if energetically possible, vibrational predissociation (VP), the presence/absence of $\mathrm{M}^{\neq} \cdot \mathrm{S}$ is measured by the probe laser (3). ${ }^{19,22,39}$ The delay time must be long enough for IVR to go to completion, and needs to be checked for each complex. A $1.1 \mu$ s delay has been empirically found to be sufficient for 1naphthol with small admolecules such as cyclopropane.

Two types of mass-selected ion signals are observed in this experiment. First, the pump produces a one-color resonant-two-photon ionization (1C-R2PI) signal of the cold M.S complex. A decrease in this pump ion signal occurs at each dump transition because the intermediate $S_{1}$ state is depopulated. This is called the dump spectrum. The time-delayed probe laser produces a second, delayed ion signal of the M.S complex, which is called the SEP-R2PI signal. If the probe laser is tuned to the $S_{0} \rightarrow S_{1}$ origin, a decrease of the SEP-R2PI signal is observed at every dump transition to a $S_{0}$ state vibration. Both this origin-probed SEP-R2PI spectrum and the dump spectrum are similar to the fluorescence emission spectrum from the pumped state (apart from optical saturation effects in the laser spectra), but with negative-going peaks. If the probe laser is tuned into the hot-band region below the $S_{0} \rightarrow S_{1}$ origin, the SEP-R2PI ion signal increases at every resonant dump laser transition to $S_{0}^{\neq}$levels, which is denoted the hot-band probed SEP-R2PI spectrum. However, as soon as the dump transition populates $S_{0}^{\neq}$levels that lie above $D_{0}\left(S_{0}\right)$, see arrow 2' in Figure 1, the M.S complex vibrationally predissociates and the hot-band probed SEPR2PI signal disappears. The $D_{0}\left(S_{0}\right)$ is thereby bracketed between the highest-energy vibrational level observed in the hot-band probed SEP-R2PI spectrum and next higher vibrational level that is observed in the dump or origin-probed SEP-R2PI (or the fluorescence emission) spectrum.

Taking into account the spectral frequency shift $\delta \tilde{\nu}$ of the $S_{0} \rightarrow S_{1}$ origin of M.S relative to that of $\mathrm{M}$, the excited-state dissociation energy $D_{0}\left(S_{1}\right)$ is obtained as $D_{0}\left(S_{1}\right)=D_{0}\left(S_{0}\right)-\delta \tilde{\nu}^{17-19,22,39}$ This relation also follows from the right-hand side of Figure 1: The vibrationless $S_{0} \rightarrow S_{1}$ excitation energy of the chromophore $\mathrm{M}$ is the energy difference between the dissociation products $\left[\mathrm{M}^{*}+\mathrm{S}\right]$ and $[\mathrm{M}(\mathrm{v}=0)+\mathrm{S}]$, as indicated above the respective dissociation limits; the vibrationless 
$S_{0} \rightarrow S_{1}$ excitation energy of the M.S complex is given by arrow 3'. The difference of these two excitation energies is the spectral shift $\delta \tilde{\nu}$. Combining the dissociation energies $D_{0}\left(S_{0}\right)$ and $D_{0}\left(S_{1}\right)$ with the vibrationless electronic excitation energies of $\mathbf{M}$ and of $\mathbf{M} \cdot \mathbf{S}$ gives a Born-Habertype energy cycle that can be re-arranged to yield the relation above.

\section{B. Experimental methods}

The 1-naphthol-cyclopropane complexes were synthesized by supersonic expansion of 1naphthol vapor (1-NpOH, Fluka, 99\%) in 1.5 bar neon carrier gas containing $0.2 \%$ cyclopropane (Sigma-Aldrich, 99\%). The naphthol was heated to $353 \mathrm{~K}$ (vapor pressure $\sim 0.5 \mathrm{mbar}$ ). Under these conditions, the maximum relative ion signals of the $1-\mathrm{NpOH} \cdot(\text { cyclopropane })_{n} n=2,3$ and 4 clusters were about 20,7 and $3 \%$ of that of the $n=1$ complex, assuming identical detection sensitivities.

The frequency-doubled pump and dump tunable dye lasers (Lambda-Physik FL2002 and FL3002) were pumped by the same frequency-doubled Nd:YAG laser (Quanta-Ray DCR3). Typically, UV pulse energies of $0.2 \pm 0.02 \mathrm{~mJ} /$ pulse and $2.2 \pm 0.2 \mathrm{~mJ} /$ pulse were used. The probe laser (Lambda Physik LPD 3000) was separately pumped by a Continuum Surelite II frequencydoubled Nd:YAG laser with a pulse energy of $0.25 \pm 0.02 \mathrm{~mJ}$ after doubling. The bandwidth of all three dye lasers is $0.3 \mathrm{~cm}^{-1}$ in the visible $(620-660 \mathrm{~nm})$. The dump wavelength was monitored by a HighFinesse WS6 wavemeter. The probe laser was delayed by $1.1 \mu$ s relative to the pump and dump laser pulses and crossed the molecular beam $\sim 1 \mathrm{~mm}$ downstream of the pump/dump lasers, corresponding to the distance traveled by the Ne supersonic molecular beam $(950 \mathrm{~m} / \mathrm{s}$ mean speed). Other experimental details were similar to those employed previously. ${ }^{17-19,22,39}$

Resonant two-photon ionization dump and probe spectra were recorded by monitoring the mass-to-charge ratio of the complex $(\mathrm{m} / \mathrm{z} 186)$ with a $1.2 \mathrm{~m}$ linear time-of-flight mass spectrometer. To identify isomeric complexes of the same composition, hole burning spectroscopy was performed. In this technique, a laser is fixed at $S_{0} \rightarrow S_{1}$ origin of an M.S isomer, causing depletion of the isomer $S_{0}$. A second laser, with a temporal delay of 100-150 ns, is scanned over the region of interest. Any bands originating from the depleted isomer ground state can easily be identified by their reduced intensities compared to the same scan without the hole-burning laser.

Single vibronic level fluorescence spectra were measured by exciting the respective $0_{0}^{0}$ band. The fluorescence emission was collected with UV quartz optics and dispersed with a SOPRA 
UHRS F1500 $1.5 \mathrm{~m}$ monochromator in second order, using $200 \mu \mathrm{m}$ slit widths, equivalent to a $0.028 \mathrm{~nm}$ bandpass; the spectra were scanned with $0.0025 \mathrm{~nm}$ step size.

\section{Theoretical Methods}

All calculations were performed using Turbomole $7.0 .{ }^{42}$ Geometry optimizations for the 1naphthol-cyclopropane complexes and the corresponding monomers were performed with the SCS-CC2 method in combination with the aug-cc-pVTZ basis set. The preference for CC2 over MP2 is due to the availability of excited state gradient methods which allow for the calculation of excited state properties. SCS-CC2 has been successfully used for ground and excited states of aromatic systems. ${ }^{43,44}$ For comparison, the structures were also optimized using the PBE0 and B3LYP density functionals in conjunction with the D3 correction with Becke-Johnson damping (PBE-D3BJ, B3LYP-D3); the def2-TZVP basis set was used with PBE0-D3 and the TZVPP basis set with B3LYP-D3. ${ }^{45-50}$

12 different starting structures were generated and then optimized using the two DFT methods. The edge isomer (denoted A, see below), is found when placing the cyclopropane at the $\mathrm{OH}$ edge of 1-naphthol both in coplanar and perpendicular alignment. The face isomers B and B' are obtained when placing cyclopropane above the 1-naphthol plane; depending on the orientation the optimizations converge to B or B'. Starting structures in which the cyclopropane is above the $\mathrm{OH}$ group of 1-naphthol optimize to the edge isomer A.

The optimization thresholds were set to $10^{-4} E_{\mathrm{h}} / a_{0}$ for the changes in the gradient and to $10^{-6} E_{\mathrm{h}}$ for changes in the energy. All structures were confirmed to be minima, without imaginary harmonic vibrational frequencies. The above mentioned optimization thresholds were sufficient to compute the intermolecular electronic binding energies to within $2 \cdot 10^{-3} \mathrm{~kJ} / \mathrm{mol}$ for 1-naphthol-cyclopropane. This was shown by running test calculations with tighter optimization thresholds of $10^{-6} E_{\mathrm{h}} / a_{0}$ for changes in the gradient and $10^{-8} E_{\mathrm{h}}$ for changes in the energy.

At the optimized SCS-CC2/aug-cc-pVTZ geometries, the intermolecular electronic binding energy $D_{e}\left(R_{\text {min,intra+inter }}\right)$ was calculated from the total electronic energy of the $\mathrm{M} \cdot \mathrm{S}$ complex, relative to the respective energies of $\mathrm{M}$ and $\mathrm{S}$ at their optimized structures, $D_{e}=\mid E_{\mathrm{M} \cdot \mathrm{S}}(\mathrm{M} \cdot \mathrm{S})-$ $E_{\mathrm{M}}(\mathrm{M})-E_{\mathrm{S}}(\mathrm{S}) \mid$. The first excited states of 1-naphthol and its cyclopropane complexes were also optimized at the SCS-CC2/aug-cc-pVTZ level with the same thresholds $\left(10^{-4} E_{\mathrm{h}} / a_{0}\right.$ and $10^{-6}$ $\left.E_{\mathrm{h}}\right)$. At each point in the excited state geometry optimization, the first five excited states were 
calculated to guarantee that the right electronic state was optimized.

The following comparative calculations were also performed: SCS-CC2/CBS(aug-cc-pV(TQ)Z), SCS-MP2/CBS(aug-cc-pV(TQ)Z), (SCS-)MP2-F12(2*B)/CBS(aug-cc-pV(TQ)Z) and CCSD(T)/ CBS(aug-cc-pV(DT)Z. Counterpoise (CP) corrections were applied for all methods except for the explicitly correlated (SCS)-MP2-F12 calculation, because it can be neglected for this level of theory. Basis set extrapolations were carried out separately for all components in the $\mathrm{CP}$ calculations, and the final $\mathrm{CP}$ correction was calculated from the $\mathrm{CBS}$ values of the underlying sub/supersystems. The PBE0-D3 interaction energy was also extrapolated to the CBS limit (def2(TQ)ZVP) and CP-corrected at the PBE0-D3/def2-TZVP optimized geometry. The Hartree-Fock part of the energy was extrapolated according to ref. 51. The conventional and F12 correlation energies were extrapolated according to Eq. (2) in ref. 52. All calculated intermolecular electronic binding energies $D_{e}$ were corrected by the change in harmonic zero-point vibrational energy, $\triangle \mathrm{ZPE}$, to yield the dissociation energies $D_{0}$.

$\triangle \mathrm{ZPE}$ is defined as $\triangle \mathrm{ZPE}=\mathrm{ZPE}_{\mathrm{M} \cdot \mathrm{S}}(\mathrm{M} \cdot \mathrm{S})-\mathrm{ZPE}_{\mathrm{M}}(\mathrm{M})-\mathrm{ZPE}_{\mathrm{S}}(\mathrm{S})$. For B3LYP-D3 and PBE0D3 the $\triangle \mathrm{ZPE}$ corrections were calculated at the corresponding levels of theory using analytical second derivatives. All dissociation energies from wave function methods were computed with $\triangle$ ZPE values obtained at the SCS-CC2/aug-cc-pVTZ level of theory using numerical second derivatives. Anharmonic corrections to the ZPEs were neglected during this study. These have been shown to be up to $15 \%$ of the harmonic $\triangle \mathrm{ZPE}$ correction. ${ }^{53}$ In our case this amounts to about $0.7 \mathrm{~kJ} / \mathrm{mol}$ or $4-5 \%$ of the experimental dissociation energies (see below). Given the high accuracy of the experimental values, anharmonic corrections are highly desirable. Unfortunately, the computational effort required for these large complexes vastly exceeds the currently available resources.

\section{RESULTS}

\section{A. Vibronic spectra of 1-Naphthol-Cyclopropane}

The mass-selected 1C-R2PI spectrum of 1-naphthol-cyclopropane is shown in Figure IV(a), and exhibits intense peaks at 31384.4 (A) and $31457.8 \mathrm{~cm}^{-1}$ (B). UV holeburning at band A results in reduced intensities at $31384.4 \mathrm{~cm}^{-1}$ and $31415.0 \mathrm{~cm}^{-1}$, see the blue dashed arrows in Figure IV(b).

The weak band at $31415.0 \mathrm{~cm}^{-1}$ above the origin is assigned to an $S_{1}$ state intermolecular vibration 
of isomer A. The hole-burning spectrum Figure IV(b) exhibits a number of unchanged bands to higher wavenumber, suggesting the presence of at least one more 1-naphthol-cyclopropane isomer. As shown in Figure IV(c), holeburning at band B reduces the intensities of seven bands (red dashed arrows) which are assigned as intermolecular vibrational excitations of isomer B at 31.2, 58.9, $63.6,67.8,76.2,85.1$ and $87.7 \mathrm{~cm}^{-1}$. Compared to bare 1- $\mathrm{NpOH}$, the electronic origin of isomer A is spectrally red shifted by $\delta \tilde{\nu}=-71.5 \mathrm{~cm}^{-1}$. The $S_{0} \rightarrow S_{1}$ origin band of isomer $\mathrm{B}$, however, is shifted slightly to the blue by $\delta \tilde{\nu}=+1.9 \mathrm{~cm}^{-1}$.

The bands marked with asterisks in Figure IV originate from 1-naphthol-(cyclopropane) ${ }_{2}$ clusters, some of which undergo prompt cyclopropane loss after 1C-R2PI and appear in the 1naphthol·cyclopropane mass channel.

\section{B. Dissociation Energies}

The hot-band probed SEP-R2PI spectrum of isomer A is shown in Figure 3(a); it was recorded with the probe laser $44 \mathrm{~cm}^{-1}$ to the red of the $0_{0}^{0}$ band of isomer A. The last positive peak appears at $1280.0 \mathrm{~cm}^{-1}$, which represents the lower limit for $D_{0}\left(S_{0}\right)$. To find the upper limit, both the dump spectrum, shown in Figure 3(b), and the single vibronic level fluorescence spectra (shown as Figure S1, supplementary material) were compared. They exhibit identical vibrational structure within the instrumental bandwidths, as shown in Figure S1. The next observed band above $1280.0 \mathrm{~cm}^{-1}$ at $1285.6 \mathrm{~cm}^{-1}$ is clearly not observed in the SEP-R2PI spectrum, Figure 3(a). Therefore, the ground state dissociation energy of isomer $\mathrm{A}$ is bracketed as $D_{0}\left(\mathrm{~S}_{0}\right)=1282.8 \pm 2.8 \mathrm{~cm}^{-1}$ or $15.35 \pm 0.03 \mathrm{~kJ} / \mathrm{mol}$.

The hot band probed SEP-R2PI spectrum of isomer B is shown in Figure 4(a); the probe laser was set $25 \mathrm{~cm}^{-1}$ to the red of the $0_{0}^{0}$ band. The highest positive band at $1407.6 \mathrm{~cm}^{-1}$ is the lower limit of $D_{0}\left(S_{0}\right)$. It is compared to the dump spectrum, Figure 4(b), which shows three weak negative-going bands, the lowest of which lies at $1427.1 \mathrm{~cm}^{-1}$, thus yielding the upper limit for $D_{0}\left(S_{0}\right)$. These bands bracket the $S_{0}$ state dissociation energy of isomer B as $D_{0}\left(S_{0}\right)=$ $1417.4 \pm 9.8 \mathrm{~cm}^{-1}$ or $16.96 \pm 0.12 \mathrm{~kJ} / \mathrm{mol}$.

In addition to the intense positive peaks, the hot-band probed SEP-R2PI spectrum in Figure 4(a) exhibits very weak negative bands that coincide with the negative-going bands in Figure 4(b). To understand the former, we note that a small and spectrally flat baseline signal is present in the hotband probed SEP-R2PI spectrum that originates from $S_{0}^{\neq}$levels that are populated by spontaneous 
fluorescence from the pumped $S_{1} ; v^{\prime}=0$ level. The weak negative peaks reflect the decrease of these fluorescence-populated $S_{0}^{\neq}$levels that occurs when the dump laser is in resonance with a predissociating $S_{0}$ state vibrational level. Such weak negative peaks have been observed in other hot-band probed SEP-R2PI spectra. ${ }^{17-19,22,39}$

The excited state dissociation energies $D_{0}\left(S_{1}\right)$ can then be determined using the electronic spectral shift $\delta \tilde{\nu}$ between the $S_{0} \rightarrow S_{1}$ origin bands of the M.S complex and of bare M, combined with the relation $D_{0}\left(S_{1}\right)=D_{0}\left(S_{0}\right)-\delta \tilde{\nu} .{ }^{17-19,22,39}$ With $\delta \tilde{\nu}=-71.5 \pm 0.5 \mathrm{~cm}^{-1}$ for isomer A and $1.9 \pm 0.5 \mathrm{~cm}^{-1}$ for isomer $\mathrm{B}$, the $D_{0}\left(S_{1}\right)$ values are determined as $1354.3 \pm 3.3 \mathrm{~cm}^{-1}$ and $1415.5 \pm 10.3 \mathrm{~cm}^{-1}$, respectively.

\section{Isomer Structures: Experiment and Theory}

The electronic spectral shift $\delta \tilde{\nu}$ between the $S_{0} \rightarrow S_{1}$ origin bands of M.S complex and of bare M provides indirect but useful structural information, as found in phenol and 1-naphthol with water, methanol, or ammonia, ${ }^{39,54}$ or phenylacetylene-amine complexes. ${ }^{55}$ The 1-NpOH.S complexes with $\mathrm{S}=\mathrm{H}_{2} \mathrm{O}, \mathrm{CH}_{3} \mathrm{OH}, \mathrm{NH}_{3}, \mathrm{C}_{6} \mathrm{H}_{6}$ exhibit hydrogen bonds from the 1-NpOH hydroxyl group to $\mathrm{S}$, and exhibit spectral red shifts $\delta \tilde{\nu}=-145,-157.9,-236.3$ and $-66.3 \mathrm{~cm}^{-1}$, respectively. Regarding the structure of $1-\mathrm{NpOH} \cdot$ benzene, a rotational coherence study strongly suggested an H-bonded structure. ${ }^{56}$ In contrast, for complexes of 1-naphthol with the adsorbate above the $\pi$ electron system such as $1-\mathrm{NpOH} \cdot \operatorname{Ar}\left(\delta \tilde{\nu}=-15 \mathrm{~cm}^{-1}\right)$ and $1-\mathrm{NpOH} \cdot \mathrm{N}_{2}\left(\delta \tilde{\nu}=-14 \mathrm{~cm}^{-1}\right)$, the shifts are much smaller.

The $\delta \tilde{\nu}=-71.5 \mathrm{~cm}^{-1}$ of isomer $\mathrm{A}$ of $1-\mathrm{NpOH} \cdot$ cyclopropane is rather similar to that of 1$\mathrm{NpOH} \cdot$ benzene $\left(-66.3 \mathrm{~cm}^{-1}\right)$, suggesting that the cyclopropane is coordinated to the $\mathrm{OH}$ group of 1-naphthol hydroxyl group, in an edge-on fashion. This will be called the edge isomer below. In isomer $\mathrm{B}$, the spectral shift $\delta \tilde{\nu}=1.9 \mathrm{~cm}^{-1}$ is small and close to that of van der Waals complexes. Isomer B is therefore likely a $\mathrm{CH} \cdots \pi$ complex with the cyclopropane on the $\pi$-face of the naphthol. It will be denoted the face isomer in the following.

In accord with the binding topologies inferred from the spectral shifts, both edge and face structures were located on the calculated potential energy surface of 1-naphthol-cyclopropane, as shown in Figure 5. However, two face minima were found: In the higher energy minimum B' shown in Figure 5(c), the cyclopropane ring is above the three naphthol ring carbons opposite the hydroxyl. In the lower (global) minimum B shown in Figure 5(d) the cyclopropane is closer 
to the lone-pairs of the electron-rich $\mathrm{O}$ atom and the cyclopropane ring is about $3.5 \AA$ above the naphthalene rings.

In the edge isomer, the naphthol $\mathrm{OH}$ group is directed towards the center of a cyclopropane $\mathrm{CC}$ bond, the complex has $C_{s}$ symmetry. The distance from the hydroxyl proton to the two proximal cyclopropane $\mathrm{C}$ atoms is $2.31 \AA$, and from the $\mathrm{H}$ atom to the midpoint of the $\mathrm{CC}$ bond is $2.18 \AA$. The $\mathrm{H}$ atom of the $\mathrm{OH}$ group is nearly $0.4 \AA$ closer to the nearest $\mathrm{CC}$ bond than the $\mathrm{H}$ atoms in the face-on isomer. This $\mathrm{OH}$ - cyclopropane bonding motif is very similar to that of the F$\mathrm{H} \cdots$ cyclopropane complex, ${ }^{29}$ and is also similar to the crystallographically observed $\mathrm{CH} \cdots \pi$ (alkene) interaction geometries. ${ }^{24}$

The B' structure can be converted to B by translating the cyclopropane in-plane across the naphthalene ring and rotating it by $\sim 60^{\circ}$. The calculated ZPE-corrected energy difference between B' and B is $1.2-1.4 \mathrm{~kJ} / \mathrm{mol}$ with the density functional methods. The calculated barrier from B' towards B is $0.20 \mathrm{~kJ} / \mathrm{mol}$. No experimental evidence for two coexisting face isomers was found, consistent with this low calculated barrier. The lowest barrier from B toward the edge isomer A is calculated as $8.9 \mathrm{~kJ} / \mathrm{mol}$ using PBE0-D3/def2-TZVP reaction path optimizations. The PBE0D3 calculated $\mathrm{B}^{\prime} \leftrightarrow \mathrm{B}$ and $\mathrm{B} \leftrightarrow \mathrm{A}$ transition structures are given in Figure S2 and Tables S15/S16 (supplemental material).

The face isomer is $135 \mathrm{~cm}^{-1}$ or $1.6 \mathrm{~kJ} / \mathrm{mol}$ more stable than the edge isomer; this is $20-25$ times $k_{B} T$ at the molecular beam temperature. Furthermore, the existence of two symmetryequivalent face minima statistically favors the face isomer by a factor of two. Nevertheless, the R2PI intensities in Figure 2(a) are almost equal. This implies that there are substantial barriers on the intermolecular potential energy surface which separate the minima, allowing the less stable isomer to be kinetically trapped as the supersonic jet expands and cools. It may also be that the catchment region (or area) that leads to the edge isomer is substantially larger than that leading to the face minima. Dipole-induced dipole interactions are of longer range than dispersive interactions, so the hydroxyl group might have a significant directing effect.

\section{Calculated Binding and Dissociation Energies}

Table I gives an overview of the calculated intermolecular dissociation energies. For the corre-

lated wave function methods the energies were calculated at the SCS-CC2/aug-cc-pVTZ optimized geometry. The binding energies $D_{e}$ were extrapolated to the basis set limit and were then corrected 
for zero-point vibrational energy as described in section II.C.

All wave function methods give a slightly larger $D_{0}$ for the face than for the edge isomer, in agreement with the experimental ordering. However, the absolute dissociation energies are too small for all wave function methods except $\operatorname{CCSD}(\mathrm{T})$ at the complete basis set limit. Note that the CCSD(T)/aug-cc-pVTZ calculations differ by 11 and $17 \%$ from the experimental $D_{0}$ values, and a basis set extrapolation is mandatory. This was done from double- and triple- $\zeta$ basis sets, denoted as aug-cc-pV(DT)Z. Since the structures were not optimized with each method, there may be systematic errors due to the geometry, but these are expected to be small.

TABLE I. Calculated dissociation energies $D_{0}$ (in $\mathrm{kJ} / \mathrm{mol}$ ) of the face and edge structures of the 1naphthol-cyclopropane complex. Values that are extrapolated to the complete basis set (CBS) limit using a two-point procedure are marked CBS.

\begin{tabular}{lcccccc}
\hline Method & Basis set & Face B & Face B & Error, \% & Edge & Error, \% \\
\hline Experiment & & & $16.96 \pm 0.12$ & & $15.35 \pm 0.03$ & \\
\hline Wave function & methods & & & & & \\
MP2-F12 & aug-cc-pV(TQ)Z, CBS & 19.83 & 21.71 & +28.0 & 15.25 & -0.7 \\
SCS-MP2 & aug-cc-pV(TQ)Z, CBS & 11.91 & 13.19 & -22.2 & 10.78 & -29.8 \\
SCS-MP2-F12 & aug-cc-pV(TQ)Z, CBS & 11.48 & 12.82 & -24.4 & 10.65 & -30.6 \\
SCS-CC2 & aug-cc-pVTZ, CP corr. & 10.84 & 12.19 & -28.1 & 10.69 & -30.4 \\
SCS-CC2 & aug-cc-pV(TQ)Z, CBS & 11.55 & 12.96 & -23.9 & 11.42 & -13.9 \\
CCSD & aug-cc-pV(DT)Z, CBS & 8.65 & 10.02 & -40.9 & 11.38 & -25.9 \\
CCSD(T) & aug-cc-pVTZ, CP corr. & 13.00 & 14.15 & -16.6 & 13.63 & -11.2 \\
CCSD(T) & aug-cc-pV(DT)Z, CBS & 14.24 & 15.93 & -6.1 & 14.92 & -2.8 \\
\hline Density functional methods & & & & & \\
PBE0-D3 & def2-(TQ)ZVP, CBS & 15.11 & 16.21 & -4.4 & 15.34 & -0.1 \\
B3LYP-D3 & TZVPP & 15.64 & 17.07 & +0.6 & 15.52 & +1.1 \\
\hline Symmetry-adapted perturbation theory & & & & & -17.0 \\
\hline $\begin{array}{l}\text { DFT-SAPT } \\
\text { S }\end{array}$ & aug-cc-pVTZ & 12.12 & 13.43 & -20.8 & 12.74 & \\
\hline
\end{tabular}

For dispersively bound structures it is crucial to take into account the $\triangle \mathrm{ZPE}$ corrections. As a calculation of full second derivatives is not possible for many high-level wave function methods such CCSD or CCSD(T), this has to be done at a lower level of theory. As CC2 is an approximation 
to $\mathrm{CCSD}$, it is often a sensible choice for correcting other coupled cluster methods. For the $\triangle \mathrm{ZPE}$ correction the spin-component-scaled variant yields similar results to unscaled CC2 with a maximum difference of $0.05 \mathrm{~kJ} / \mathrm{mol}$. When the (SCS-)CC2 $\triangle \mathrm{ZPE}$ corrections are compared to those obtained with PBE0-D3, the difference can be as large as $1.4 \mathrm{~kJ} / \mathrm{mol}$. Interestingly, B3LYP-D3 yields $\triangle \mathrm{ZPE}$ corrections similar to those obtained with (SCS-)CC2.

The dispersion-corrected density functional methods performed better than their wave function counterparts, which may be partly because the geometries and the $\triangle$ ZPEs were calculated with the same methods. The popular hybrid B3LYP functional with D3 dispersion correction performed remarkably well for this complex. It performs less well with other, particularly larger, dispersiondominated complexes, as will be reported elsewhere. The PBE0 functional in conjunction with a D3 correction yields gives results at $\operatorname{CCSD}(\mathrm{T})$ quality in a fraction of time. As the basis set dependence and the basis set superposition errors are much less severe for DFT methods, quantitative results can already be obtained with a triple- $\zeta$ basis set level. DFT-SAPT however performed no better than the wave function methods.

MP2-F12/CBS was capable of correctly describing the edge isomer where the bonding is partially electrostatic. The overestimate of the binding energy of the face isomer is no doubt due to the well known uncoupled description of dispersive interactions. ${ }^{57}$ However, when a truncated basis is used, fortuitous error cancelation can occur, making (SCS)-MP2/CC2 methods viable alternatives. At the CBS limit, however, spin-component-scaled methods underestimate dispersion interaction by as much as the pure MP2 overestimates them.

The observation of two isomers raises questions about differences in intermolecular interactions, particularly the role of dispersion. Since the dispersive interactions are added explicitly to density functional methods, it is easy to determine the dispersion contribution. In the case of the B3LYP-D3 method, Table II shows the D3 contribution to $D_{e}$ for the isomers. Not surprisingly, the binding energy of the face isomer is completely dispersion dominated, without the D3 correction this isomer would be unbound. The fact that the cyclopropane is adsorbed nearly flat on the naphthalene face means that many atoms are relatively close, the total D3 correction is almost $27 \mathrm{~kJ} / \mathrm{mol}$. The edge isomer experiences less than half as much dispersion stabilization as the face isomer, since the cyclopropane is rather far from the bulk of the naphthalene moiety. However, the dispersive contribution is still twice that of the non-dispersive binding energy of $6.5 \mathrm{~kJ} / \mathrm{mol}$. The latter presumably includes an electrostatic component between the hydroxyl dipole and the corresponding induced dipole in the cyclopropane. 
TABLE II. Dispersion contribution to the $D_{e}\left(S_{0}\right)$ of 1-naphthol-cyclopropane at the B3LYP-D3/TZVPP level (in $\mathrm{kJ} / \mathrm{mol}$ ). The first two columns are the $D_{e}$ with and without the D3 correction, at the B3LYP-D3 equilibrium geometry. The last column is their difference.

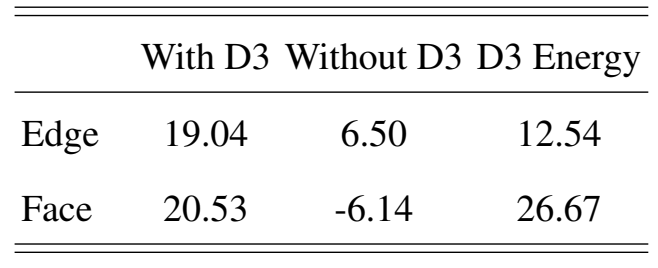

With both the PBE0-D3 and SCS-CC2 methods, a larger red-shift $\delta \tilde{\nu}$ is predicted for the edge isomer than for the face isomer, in qualitative agreement with experiment. However, the PBE0-D3 spectral shifts are much too large, possibly indicating a systematic limitation of the method for the calculation of the excited-state binding energy. The SCS-CC2/aug-cc-pVTZ spectral shifts are very close to the experimental ones, differing by only -4 and $-14 \mathrm{~cm}^{-1}$.

TABLE III. $S_{0} \rightarrow S_{1}$ spectral shifts, $\delta \tilde{\nu}\left(\right.$ in $\mathrm{cm}^{-1}$ ), calculated with the SCS-CC2 and PBE0-D3 methods.

\begin{tabular}{|c|c|c|}
\hline & Face $(\mathrm{B})$ & Edge (A) \\
\hline Experiment & +1.9 & -71.5 \\
\hline PBE0/def2-TZVP & -148.7 & -329.3 \\
\hline SCS-CC2/aug-cc-pVTZ & -2.3 & -85.9 \\
\hline
\end{tabular}

\section{CONCLUSIONS}

The 1-naphthol-cyclopropane complex was formed in a supersonic jet expansion. Two isomers were observed by resonant two-photon ionization spectroscopy and confirmed by UV/UVholeburning. The spectral shifts of the respective $S_{0} \rightarrow S_{1}$ electronic origins combined with calculations lead to the assignment of a face isomer B, which exhibits a spectral blue shift of $\delta \nu=+2 \mathrm{~cm}^{-1}$ and an edge isomer A with a spectral red shift of $\delta \nu=-72 \mathrm{~cm}^{-1}$. In the former the cyclopropane is dispersively bound to the $\pi$-electron system of naphthalene, in the latter the intermolecular binding is between the naphthol $\mathrm{OH}$ and a cyclopropane $\mathrm{CC}$ bond.

Using the stimulated-emission pumping resonant two-photon ionization (SEP-R2PI) method, ${ }^{9,17,18}$ the intermolecular dissociation energies $D_{0}\left(S_{0}\right)$ of both isomers was bracketed within very narrow limits. The dissociation energy of the edge isomer (A) is $15.35 \pm 0.03 \mathrm{~kJ} / \mathrm{mol}$, that of the face 
isomer (B) is $16.96 \pm 0.12 \mathrm{~kJ} / \mathrm{mol}$, or about $10 \%$ larger. The relative uncertainties are 0.2 and $0.7 \%$, respectively. Such accuracies have so far only been reported for small H-bonded complexes, such as the dimers and trimers of $\mathrm{HF}, \mathrm{H}_{2} \mathrm{O}$ and $\mathrm{HCl}^{30-32,58}$

Both correlated wave function and dispersion-corrected density functional calculations predict the face and edge isomers. There are two calculated face structures, B and B', with a low barrier between them; we observe only one face isomer. While the edge/face ordering of the dissociation energies predicted by the wave function methods was correct, the energies themselves generally were not satisfactory, the errors are typically larger than $\pm 10 \%$ and range up to $\pm 30 \%$. Only $\operatorname{CCSD}(\mathrm{T})$ extrapolated to the complete basis set limit is capable of a satisfactory description of this complex, providing $D_{0}$ values that differ from experiment by $3-6 \%$. The major drawback of $\operatorname{CCSD}(\mathrm{T})$ is that this otherwise highly precise method needs to be ZPE corrected at a lower level of theory. In contrast, the computationally much faster PBE0-D3 and especially the B3LYPD3/TZVPP method provided dissociation energies accurate to within a few percent (PBE0-D3) and within $\sim 1 \%(B 3 L Y P-D 3)$.

At the B3LYP-D3 level, the stabilization of the face isomer is completely dominated by London dispersion interactions. When omitting the D3 corrections this isomer would not be bound. The dispersion contribution to the binding of the edge isomer also large, being two thirds of the total dissociation energy.

We hope that these high precision measurements on computationally tractable dispersion- dominated clusters with different isomers can act as useful reference data for further development of theory and provide insight into dispersion itself.

\section{SUPPLEMENTARY MATERIAL}

See supplementary material for comparison of SVLF and dump spectra, calculated B' $\leftrightarrow$ B and $\mathrm{B} \leftrightarrow \mathrm{A}$ transition structures, calculated $\triangle \mathrm{ZPE}$ corrections and 29 tables with Cartesian coordinates of the structures of 1-naphthol, cyclopropane and of the A, B and B' isomers of 1naphthol-cyclopropane, optimized with the SCS-CC2, PBE0-D3 and B3LYP-D3 methods. 


\section{ACKNOWLEDGEMENTS}

We thank the Swiss National Science Foundation (SNSF) and the Deutsche Forschungsgemeinschaft (DFG) for financial support through the Priority Programme SPP 1807 "Control of London dispersion interactions in molecular chemistry" (grants DFG KL 721/5-1 and SNSF 200021E160404).

* leutwyler@dcb.unibe.ch

$\dagger{ }^{3}$ Current address: Department of Chemistry, IIT Hyderabad, Kandi, Sangareddy-502285, Telangana, India; ${ }^{4}$ Current address: LERMA, Sorbonne Universités, UPMC Univ. Paris 06, Observatoire de Paris, PSL Research University, CNRS, F-75252, Paris, France

1 M. Nishio, M. Hirota, and Y. UMEZAWA, The CH/ $\pi$ Interaction: Evidence, Nature, and Consequences, volume 21, Wiley-VCH, 1998.

2 G. R. Desiraju and T. Steiner, The Weak Hydrogen Bond in Structural Chemistry and Biology, Oxford University Press, 1999.

3 T. Steiner, Angew. Chemie Int. Ed. 41, 48 (2002).

4 Y. Umezawa, S. Tsuboyama, K. Honda, J. Uzawa, and M. Nishio, Bull. Chem. Soc. Jpn. 71, 1207 (1998).

5 S. Tsuzuki, K. Honda, A. Fuji, T. Uchimaru, and M. Mikami, Phys. Chem. Chem. Phys. 10, 2860 (2008).

6 S. Tsuzuki and A. Fujil, Phys. Chem. Chem. Phys. 10, 2584 (2008).

7 A. Fujit, H. Hayashi, J. W. Park, T. Kazama, N. Mikami, and S. Tsuzuki, Phys. Chem. Chem. Phys. 13, 14131 (2011).

8 Y. UMeZAwA and M. Nishio, Bioorganic \& Medicinal Chemistry 8, 2643 (2000).

9 J. A. Frey, C. Holzer, W. Klopper, and S. Leutwyler, Chem. Rev. 116, 5614 (2016).

10 R. K. SAmpson and W. D. Lawrance, Aust. J. Chem. 56, 275 (2003).

11 S. M. Bellm, J. R. Gascooke, and W. D. Lawrance, Chem. Phy. Lett. 330, 103 (2000).

12 S. M. Bellm, R. J. Moulds, and W. D. Lawrance, J. Chem. Phys. 115, 10709 (2001).

13 J. Černý, X. Tong, P. Hobza, and K. Müller-Dethlefs, J. Chem. Phys. 128, 114319 (2008). 
14 F. Mazzoni, M. Becucci, J. Rezac, D. Nachtigallová, F. Michels, P. Hobza, and K. Müller-Dethlefs, Phys. Chem. Chem. Phys. 17, 12530 (2015).

15 J. E. Braun, T. L. Grebner, and H. J. Neusser, J. Phys. Chem. A 102, 3273 (1998).

16 T. L. Grebner, R. Stumpf, and H. J. Neusser, Int. J. Mass Spectrom. Ion Processes 167/168, 649 (1997).

17 T. Bürgi, T. Droz, and S. Leutwyler, Chem. Phys. Lett. 225, 351 (1994).

18 T. Droz, T. Bürgi, and S. Leutwyler, J. Chem. Phys. 103, 4035 (1995).

19 T. Bürgi, T. Droz, and S. Leutwyler, J. Chem. Phys. 103, 7228 (1995).

20 K. Shibasaki, A. Fujit, N. Mikami, and S. Tsuzuki, J. Phys. Chem. A 110, 4397 (2006).

21 K. Shibasaki, A. Fujit, N. Mikami, and S. Tsuzuki, J. Phys. Chem. A 111, 753 (2007).

22 C. Wickleder, T. Droz, T. Bürgi, and S. Leutwyler, Chem. Phys. Lett. 264, 257 (1997).

23 J. Gauss, D. Cremer, and J. F. Stanton, J. Phys. Chem. A 104, 1319 (2000).

24 F. H. Allen, J. Lommerse, V. Hoy, J. Howard, and G. Desiraju, Acta Cryst. B 52, 734 (1996).

25 D. S. Kummli, H. M. Frey, M. Keller, and S. Leutwyler, J. Chem. Phys. 123, 054308 (2005).

26 C. R. Groom, I. J. Bruno, M. P. Lightfoot, and S. C. Ward, Acta Cryst. B72, 171 (2016).

27 P. von R. Schleyer, D. S. Trifan, and R. BACskai, J. Am. Chem. Soc. 80, 6691 (1958).

28 L. Joris, P. v. R. Schleyer, and R. Gleiter, J. Am. Chem. Soc. 90, 327 (1968).

29 L. Buxton, P. Aldrich, J. Shea, A. Legon, and W. Flygare, J. Chem. Phys. 75, 2681 (1981).

30 B. E. Rocher-Casterline, L. C. $\mathrm{CH}^{\prime}$ NG, A. K. Mollner, and H. Reisler, J. Chem. Phys 134, 211101 (2011).

31 L. C. Ch́ng, A. K. Samanta, G. Czakó, J. M. Bowman, and H. Reisler, J. Am. Chem. Soc. 134, 15430 (2012).

32 A. K. Samanta, G. Czakó, Y. Wang, J. S. Mancini, J. M. Bowman, and H. Reisler, Acc. Chem. Res. 47, 2700 (2014).

33 R. Leist, J. Frey, P. Ottiger, H.-M. Frey, S. Leutwyler, R. Bachorz, and W. Klopper, Angew. Chem. Int. Ed. 46, 7449 (2007).

34 R. A. Bachorz, F. A. Bischoff, S. Höfener, W. Klopper, P. Ottiger, R. Leist, J. A. Frey, and S. Leutwyler, Phys. Chem. Chem. Phys. 10, 2758 (2008).

35 T. Schwabe and S. Grimme, Acc. Chem. Res. 41, 569 (2008).

36 S. Haldar, R. Gnanasekaran, and P. Hobza, Phys. Chem. Chem. Phys. 17, 26645 (2015). 
37 A. K. Samanta, Y. Wang, J. S. Mancini, J. M. Bowman, and H. H. Reisler, Chem. Rev. 116, 4913 (2016).

38 H.-L. DAI and R. W. FIELD, Molecular Dynamics and Spectroscopy by Stimulated Emission Pumping, World Scientific, Singapore, 1995.

39 T. BÜrgi, T. Droz, and S. Leutwyler, Chem. Phys. Lett. 246, 291 (1995).

40 T. Droz, T. Bürgi, and S. Leutwyler, Ber. Bunsenges. Phys. Chem. 99, 429 (1995).

41 C. Wickleder, D. Henseler, and S. Leutwyler, J. Chem. Phys. 116, 1850 (2002).

42 Turbomole V7.0, 2015, a development of Universität Karlsruhe (TH) and Forschungszentrum Karlsruhe GmbH, 1989-2007, TURBOMOLE GmbH, since 2007; available from http://www.turbomole.com.

43 N. O. C. Winter, N. K. Graf, S. Leutwyler, and C. Hättig, Phys. Chem. Chem. Phys. 15, 6623 (2013).

44 A. Hellweg, S. A. Grün, and C. Hëttig, Phys. Chem. Chem. Phys. 10, 4119 (2008).

45 J. P. Perdew, K. Burke, and M. Ernzerhof, Phys. Rev. Lett. 77, 3865 (1996).

46 C. Adamo and V. Barone, J. Chem. Phys. 110, 6158 (1999).

47 S. Grimme, J. Antony, S. Ehrlich, and H. Krieg, J. Chem. Phys. 132, 154104 (2010).

48 F. Weigend and R. Ahlrichs, Phys. Chem. Chem. Phys. 7, 3297 (2005).

49 D. E. Woon and T. H. Dunning JR., J. Chem. Phys. 103, 4572 (1995).

50 S. Grimme, A. Hansen, C. Bannwarth, and J. G. Brandenburg, Chem. Rev. 116, 5104 (2016).

51 D. G. Truhlar, Chem. Phys. Lett. 294, 45 (1998).

52 J. G. Hill, K. A. Peterson, G. Knizia, and H.-J. Werner, J. Chem. Phys. 131, 194105 (2009).

53 K. Szalewicz and B. Jeziorski, J. Chem. Phys. 109, 1198 (1998).

54 A. Fujit, T. Ebata, and N. Mikami, J. Phys. Chem. A 106, 8554 (2002).

55 S. Maity, G. N. Patwari, S. Karthikeyan, and K. S. Kim, Phys. Chem. Chem. Phys. 12, 6150 (2010).

56 P. M. FELKer and A. H. ZEwAIL, Molecular Structures from Ultrafast Coherence Spectroscopy, in Femtosecond Chemistry, edited by J. MANZ and L. Woste, pp. 193-260, Wiley-VCH Verlag GmbH, Weinheim, Germany, 2008.

57 K. E. Riley, M. PitoňÁK, P. JurečKA, and P. HobZA, Chem. Rev. 110, 5023 (2010).

58 L. Oudejans and R. E. Miller, Annu. Rev. Phys. Chem. 52, 607 (2001). 


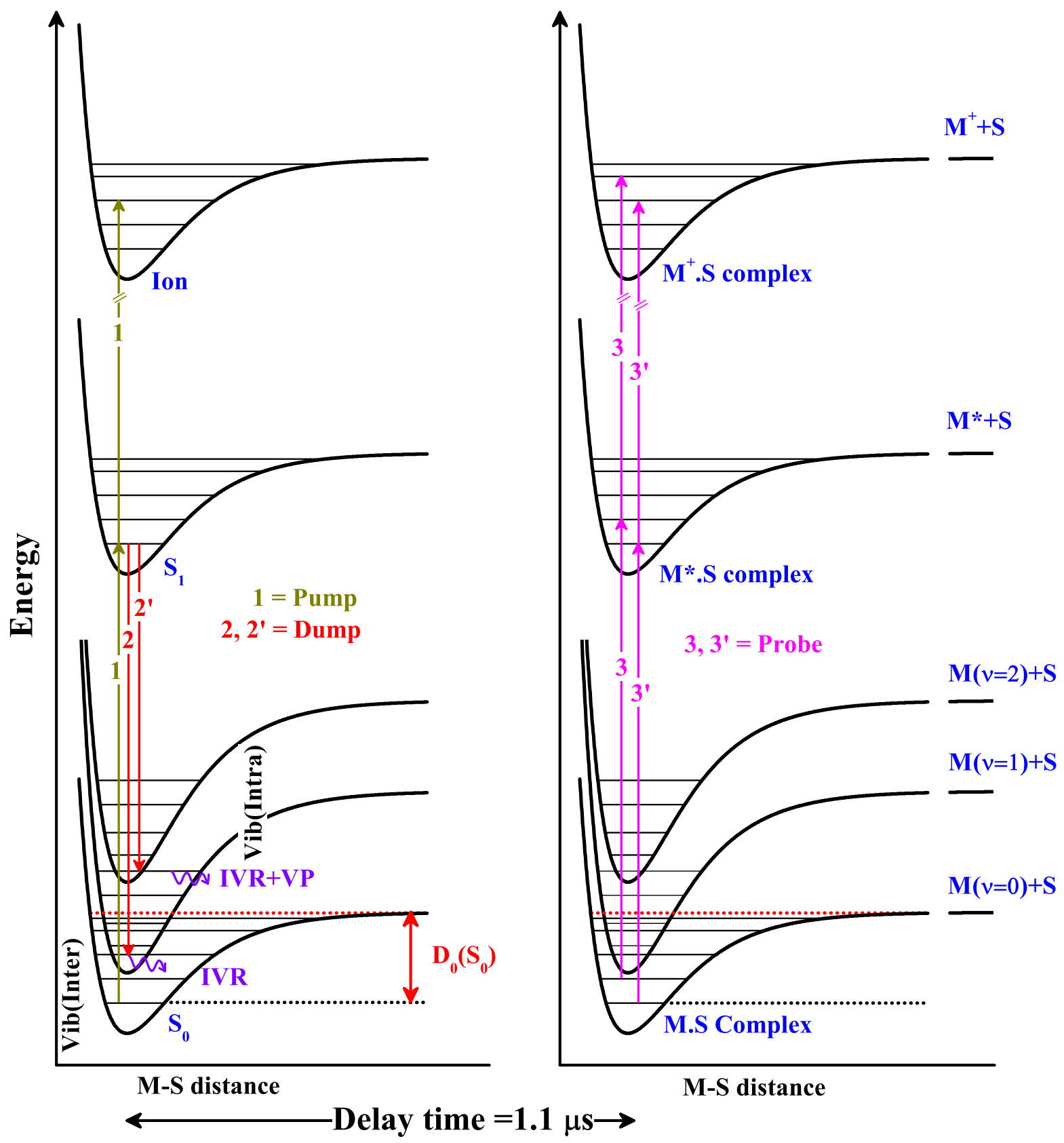

FIG. 1. Schematic level diagram of the SEP-R2PI experiment applied to M.S complex. ${ }^{9}$ The potential energy curves indicate the $S_{0}, S_{1}$ and ion ground-state potentials plotted against the M $\cdots \mathrm{S}$ distance $(\mathrm{M} \cdots \mathrm{S}$ stretching coordinate). The intramolecular vibrational levels of $\mathrm{M}$ are indicated by vertically shifted potential curves, drawn for the $S_{0}$ state only. The pump/dump steps take place nearly simultaneously, the two-photon ionization probe pulse is applied $1.1 \mu$ s later. IVR=intramolecular vibrational redistribution, $\mathrm{VP}=$ vibrational predissociation 


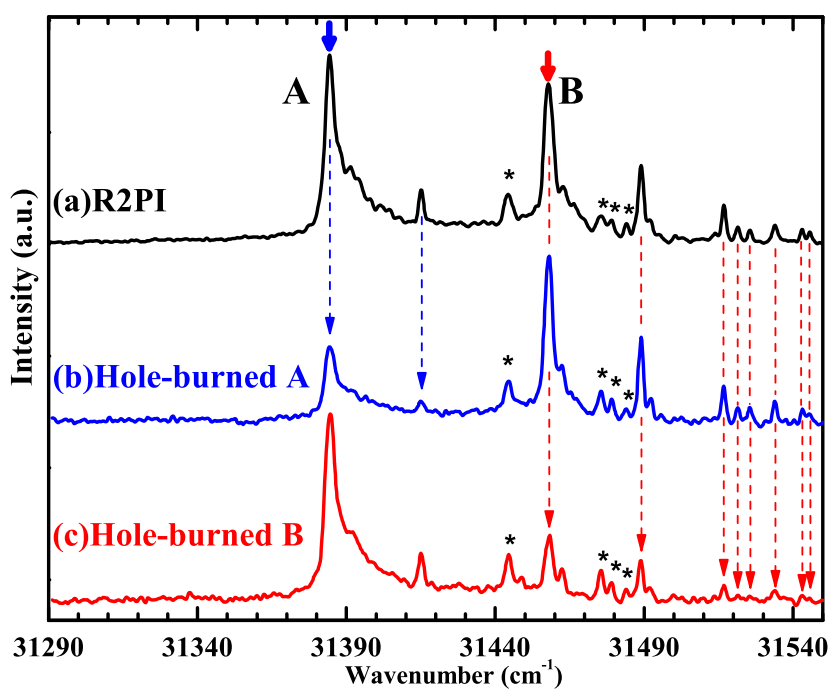

FIG. 2. (a) One-color resonant-two-photon ionization spectra of 1-naphthol-cyclopropane complexes. Peaks marked as A (at $31384.4 \mathrm{~cm}^{-1}$ ) and B (at $31457.8 \mathrm{~cm}^{-1}$ ) are the $S_{0} \rightarrow S_{1}$ electronic origin transitions of two different 1-naphthol-cyclopropane isomers. Spectra (b) and (c) depict holeburning spectra recorded with the burning laser at the origins of A and B, respectively. Arrows indicate peaks with reduced intensity. Bands with unchanged intensities marked by $*$ originate from 1-naphthol-(cyclopropane) $)_{2}$ clusters. 


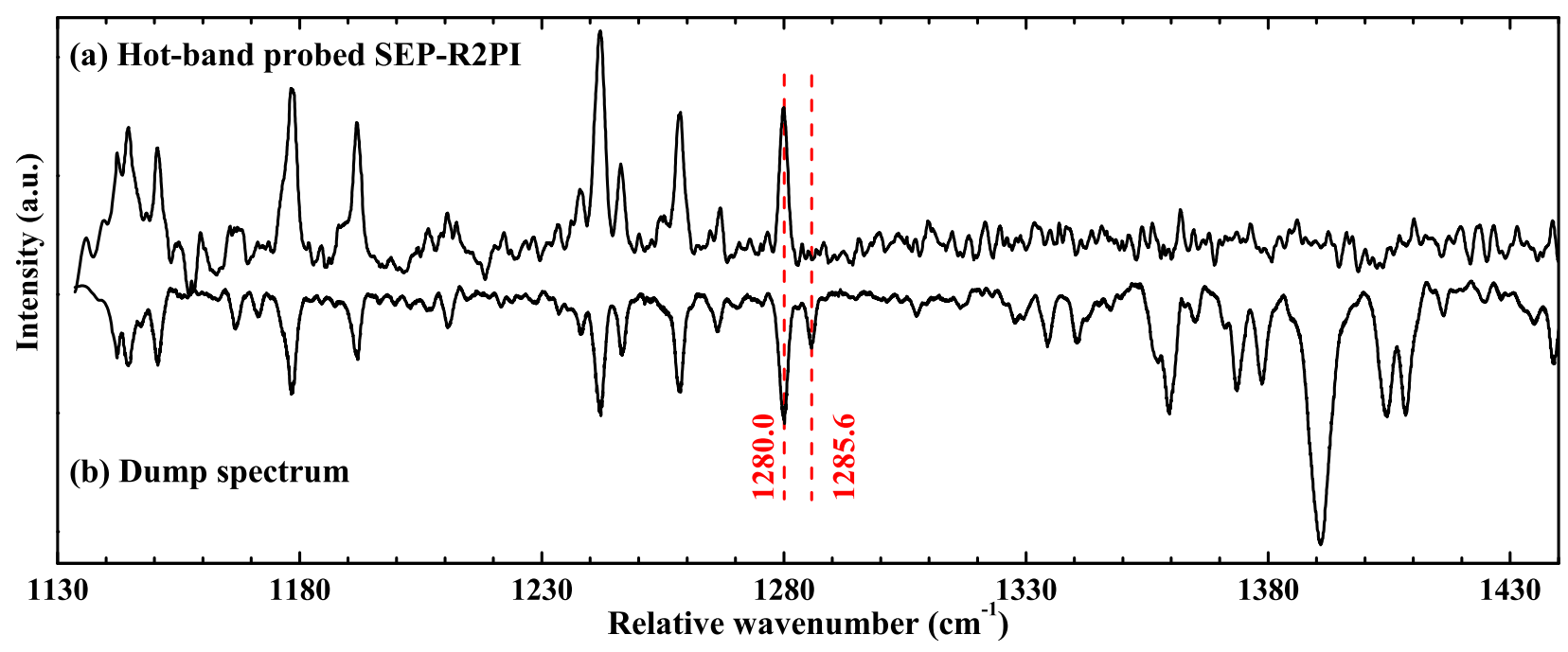

FIG. 3. (a) Hot band $\left(0_{0}^{0}-44 \mathrm{~cm}^{-1}\right)$ probed SEP-R2PI and (b) dump spectrum of isomer A of 1naphthol-cyclopropane. The $D_{0}\left(S_{0}\right)$ is bracketed by the two dashed lines at 1280.0 and $1285.6 \mathrm{~cm}^{-1}$. The wavenumber scale is the difference between the pump frequency at the isomer A $0_{0}^{0}$ band $\left(31384.4 \mathrm{~cm}^{-1}\right)$ and the dump laser frequency. 


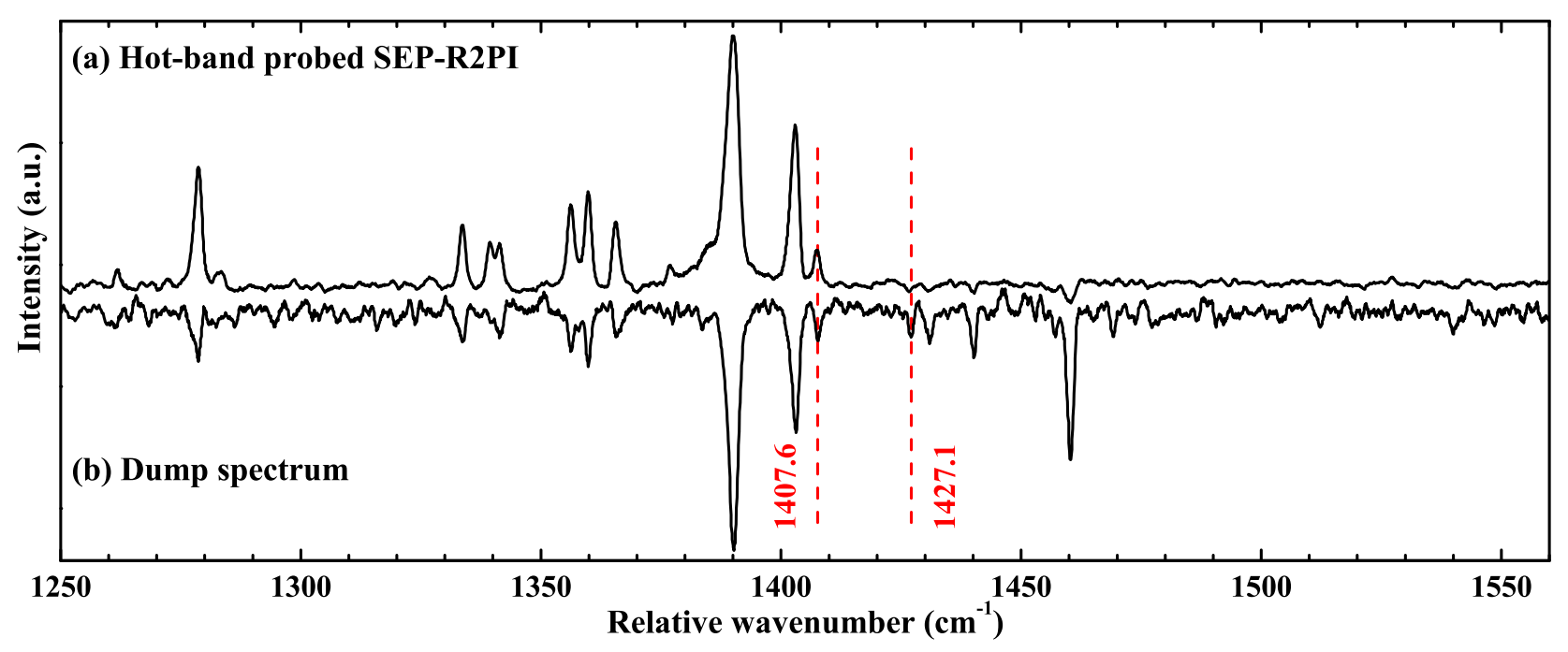

FIG. 4. (a) Hot band $\left(0_{0}^{0}-25 \mathrm{~cm}^{-1}\right)$ probed SEP-R2PI and (b) dump spectra of 1-naphthol-cyclopropane, isomer B. The $D_{0}\left(S_{0}\right)$ is bracketed within the dotted lines at 1407.6 and $1427.1 \mathrm{~cm}^{-1}$. The $\mathrm{x}$-axis wavenumber scale is the difference between the pump frequency at the isomer $\mathrm{B} 0_{0}^{0}$ band $\left(31457.8 \mathrm{~cm}^{-1}\right)$ and the dump laser frequency. 


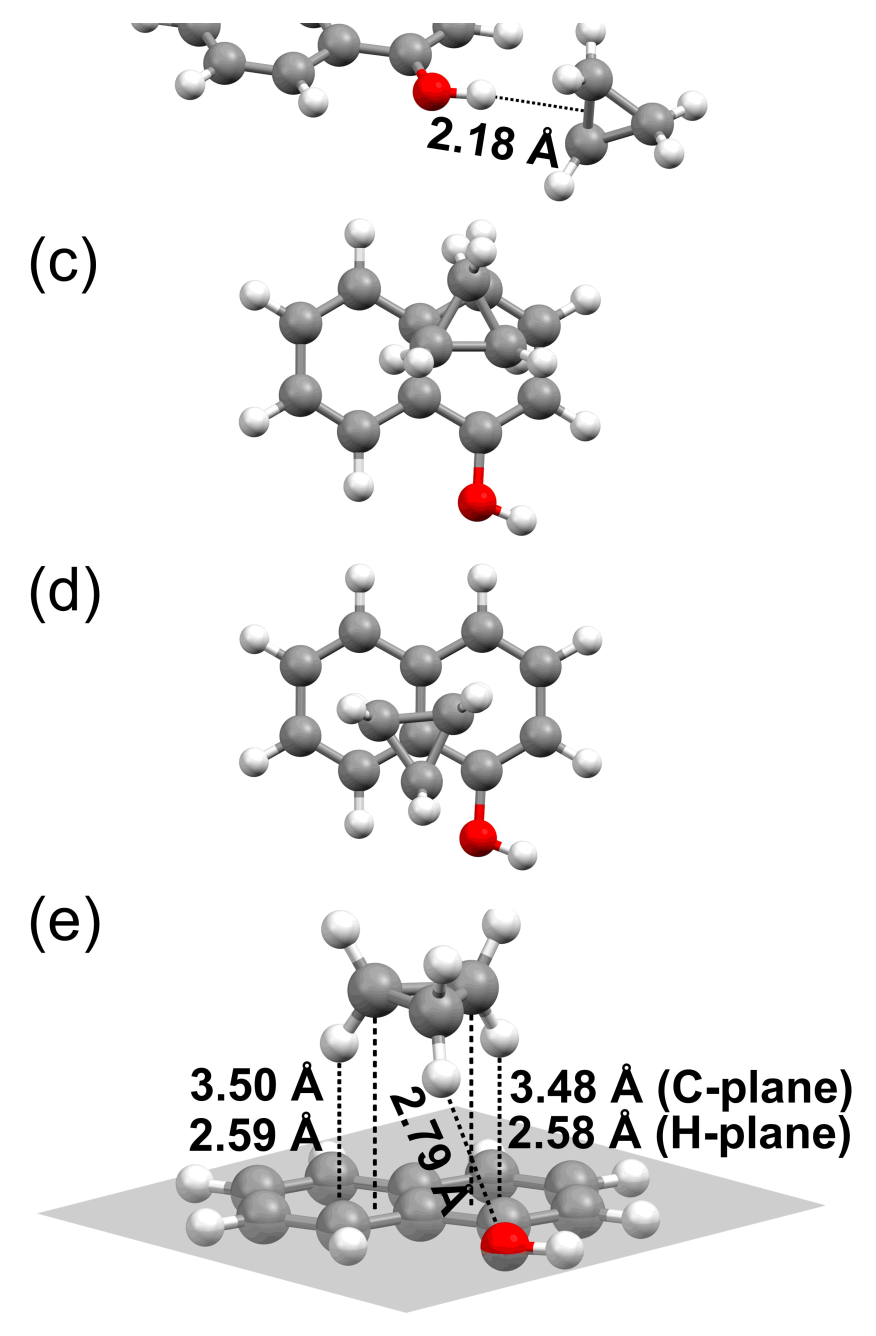

FIG. 5. (a) Edge isomer, top view (b) edge isomer, side view (c) face isomer B', top view (d) face isomer B, top view (e) face isomer B, side view. All structures optimized at the SCS-CC2/aug-cc-pVTZ level . 


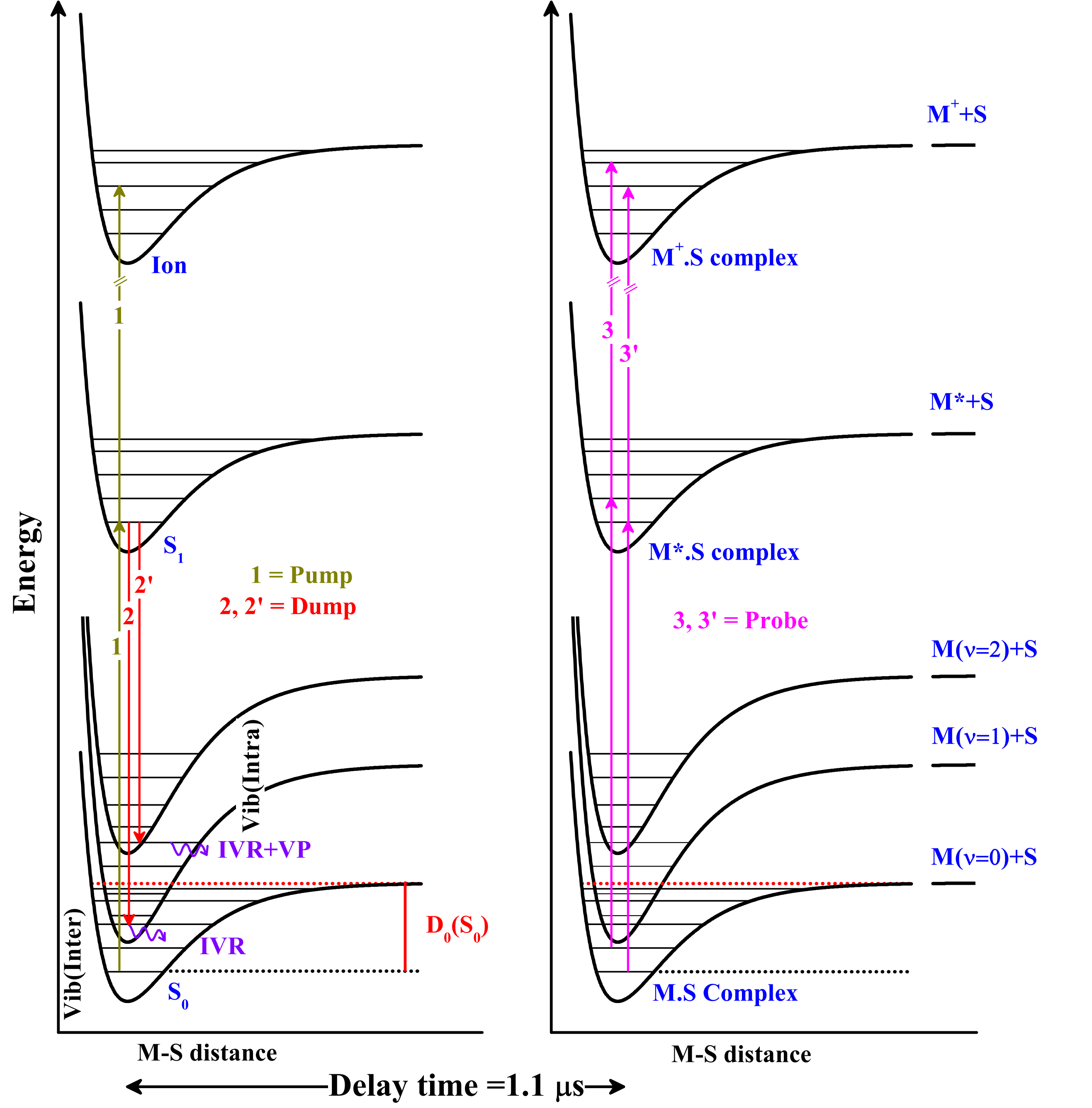




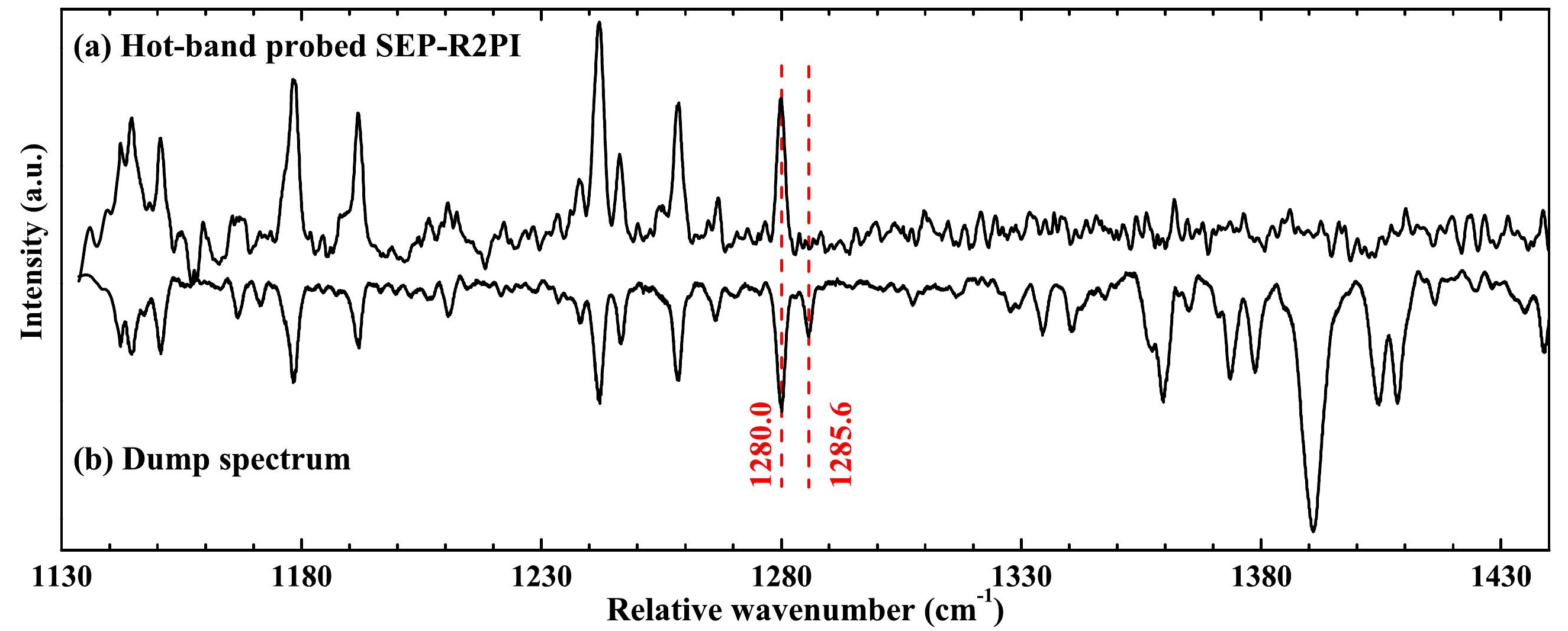


